\title{
Uso de redes sociales y rendimiento académico en estudiantes universitarios, Chachapoyas, Perú
}

\section{Use of social networks and academic performance in university students, Chachapoyas, Peru}

\author{
Tania Rocío Escobedo Tafur ${ }^{1}$, Yshoner Antonio Silva Díaz
}

\begin{abstract}
RESUMEN
El trabajo de investigación tuvo como objetivo general determinar la relación que existe entre el uso de las redes sociales y el rendimiento académico en estudiantes de Enfermería de la Universidad Nacional Toribio Rodríguez de Mendoza de Amazonas (UNTRM), Chachapoyas, 2019. La investigación fue de enfoque cuantitativo, estudio de nivel relacional, prospectivo; la muestra estuvo compuesto por 53 estudiantes del I-V ciclo de Enfermería, siguiendo un muestreo probabilístico aleatorio simple, el instrumento aplicado fue el Cuestionario de Uso de Redes Sociales y el Cuestionario de Rendimiento Académico. Del 100\% (53) de estudiantes, el 26.4\% (14) siempre utilizan las redes sociales y tienen un rendimiento académico malo, así mismo el 45.3\% (24) siempre utilizan las redes sociales y tienen un rendimiento académico regular. Por otro lado el 3.8\% (2) a veces utilizan las redes sociales y presentan un rendimiento académico regular; sin embargo el 24.5\% (13) a veces utilizan las redes sociales y tienen un rendimiento académico bueno. Para la relación de las variables se utilizó chi- cuadrado, obteniendo el valor de 43.9 calculado y 0.297 crítico, con dos grados de libertad, con un nivel de significancia de 0.05 , comprobando así que $V_{1}$ influye en $V_{2}$. $(p>0.05)$. En conclusión existe relación significativa entre $V_{1}$ y $V_{2}$ en los universitarios de Enfermería del I al V ciclo de la UNTRM, Chachapoyas.
\end{abstract}

Palabras clave: Redes sociales, estudiantes, rendimiento académico

\begin{abstract}
The general objective of de research work was to determine the relationship between the use of social networks and academic performance in nursing students, Universidad Nacional Toribio Rodríguez de Mendoza de Amazonas (UNTRM), Chachapoyas- 2019. The study of relational level, quantitative approach, prospective; the simple book consisted of 53 students of the I-V cycle of Nursing, following a simple random probabilistic sampling, the instrument applied was the Use of Social Networks Questionnaire and of Academic Performance. Of the $100 \%$ (53) of students, $26.4 \%$ (14) always use social networks and have a poor academic performance, and $45.3 \%$ (24) always use social networks and have a regular academic performance. On the other hand, 3.8\% (2) sometimes use social networks and have a regular academic performance; however, 24.5\% (13) sometimes use social networks and have a good academic performance. For the relation of de variables the chi-square is handled, obtaining the value of 43.9 calculated and 0.297 critical, whit two degrees of freedom, with a significance level of 0.05 , thus proving that $\mathrm{V}_{1}$ influences $\mathrm{V}_{2}(\mathrm{p}>0.05)$. In conclusion: There is a significant relationship between $\mathrm{V}_{1}$ and $\mathrm{V}_{2}$ in the university students of Nursing from 1 st to 5 th cycle of the UNTRM, Chachapoyas.
\end{abstract}

Keywords: Social networks, students, academic performance

\footnotetext{
Bachiller en Enfermería; Universidad Nacional Toribio Rodríguez de Mendoza de Amazonas. Correo electrónico: taniaescobedo97@gmail.com.

${ }^{2}$ Doctor. Director del Departamento Académico de Salud Pública de la Facultad de Ciencias de la Salud. Correo electrónico: yshoner.silva@untrm.edu.pe
} 


\section{INTRODUCCIÓN}

El internet ha generado un gran impacto en el mundo tecnológico, ya que se ha convertido en una gran herramienta, permitiendo la interacción de personas, sin necesidad de compartir un mismo espacio para la realización de diferentes actividades.

El acceso a internet a través de sus diferentes plataformas, dispositivos y puntos de conexión ofrece muchas alternativas para facilitar algunas actividades diarias, sin embargo, mantenerse conectados por mucho tiempo, conlleva a cosas negativas, el uso apropiado de internet dependerá de la persona y el fin que busque. (Saavedra, 2019, p. 5)

Las redes sociales forman parte de una plataforma de internet, utilizado por toda la población y los diferentes grupos etáreos, principalmente los jóvenes, pues a través de una pantalla, convierten su actuar en solo texto escrito. En la sociedad ha repercutido en los entornos de formación, no solo para tareas, sino a manera de materiales, para compartir información y establecer ambientes de trabajo. Ante la presencia inevitable de las redes sociales, el nivel de aprendizaje puede encontrarse bajo por no contar con buenas prácticas para su utilización (Marín y Cabero, 2019, p. 22).

En Latinoamérica, la plataforma de internet más utilizada es la red social facebook, pues sirve para compartir contenido, seguidamente por Instagram. Así mismo, el dispositivo más utilizado es la computadora, ya que la población que se encuentra en una oficina también acceden a las redes sociales, luego está el móvil, el cual es un dispositivo al alcance de todos (El Peruano, 2020, s/p).

El rendimiento académico tiene una definición compleja, se refiere al nivel cognitivo, habilidades adquiridas a través de información, expresados en calificaciones, donde una persona con amplio conocimiento en el tema, realiza la observación y es el encargado en brindar dicha calificación.

Según Zapata et al., el rendimiento académico mide los conocimientos construidos a través de la etapa de aprendizaje, construyendo habilidades y nuevos conocimientos, demostrando así la capacidad de comprensión de una persona. (Ibarra, J. et al. 2019)

Según Pizarro (1985), el rendimiento académico mide las capacidades que se puede manifestar, a través de resultados de un proceso de formación. (Hernández, G., y Castro, A. 2014).

El uso excesivo del internet genera un resultado negativo en cuanto al desempeño académico de los universitarios. Los hábitos de estudio y el entretenimiento sano pasan a segundo plano cuando las redes sociales están al alcance de los estudiantes, pues el mayor tiempo se encuentran conectados a una red de internet. (Saavedra, 2019, p.3).
La correlación entre las variables, se puede analizar desde diferentes opiniones, la tecnología puede ser muy útil, pues brinda información y se convierte en fuente de conocimiento para las diferentes actividades estudiantiles, sin embargo también tiene un impacto negativo, pues las diferentes páginas y plataformas se usan de manera indiscriminada, en busca de entretenimiento malo, así el tiempo dedicado al estudio y los resultados académicos, no son nada satisfactorios.

Considerando que el internet se ha integrado a la vida diaria de las personas, existen diferentes páginas de internet que buscan atraer más individuos para compartir contenidos, interactuar y crear grupos con intereses similares, de esta manera interviene en el desempeño de los estudiantes generando preocupación, pues el estudiante no está tomando una conciencia al priorizar los estudios, pues encontrarse frente a un dispositivo que favorezca su conexión a una red de internet, es más importante que realizar sus actividades académicas.

Por tanto el objetivo general fue determinar la relación entre la frecuencia del uso de las redes sociales y el rendimiento académico de los estudiantes de Enfermería, UNTRM, Chachapoyas, Perú, 2019.

\section{MATERIAL Y MÉTODO}

\subsection{Tipo de investigación}

La investigación fue de enfoque cuantitativo; de nivel relacional; observacional; prospectivo; transversal y analítico. El método a utilizar fue el método hipotético deductivo. (Supo, 2015. pp. 2 - 16), de enfoque cuantitativo.

\section{Diseño de investigación}

Se hizo uso del esquema relacional, quedando el diagrama de siguiente forma:

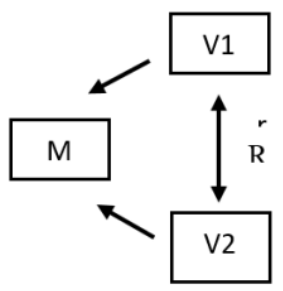

Donde:

M: Muestra

V1: Redes sociales

V2: Rendimiento académico

R: Variable de relación

\subsection{Población, muestra}

\subsubsection{Población}

Constó de 122 estudiantes del I al V ciclo, del 
semestre 2019-I, de Enfermería, UNTRM, Chachapoyas.

\subsubsection{Muestra}

La muestra utilizada para nuestra investigación fue de 53 alumnos de Enfermería.

Se utilizó la siguiente fórmula:

$$
n=\frac{Z^{2}(p)(q)(N)}{E^{2}(N-1)+Z^{2}(p)(q)}
$$

En donde:

$\mathrm{n}=$ tamaño de la muestra: 93

$\mathrm{Z}=$ nivel de confianza: $95 \%$ (en el área bajo la curva normal corresponde a 1.96: $95 / 2=0.47 .50 / 100=$ $0.4750)$

$\mathrm{p}=$ Probabilidad a favor: $50 \%(0.5)$

$\mathrm{q}=$ Probabilidad en contra: $50 \%(0.5)$

$\mathrm{N}=$ Universo: 122 estudiantes de Enfermería

$\mathrm{E}=$ error de estimación: $5 \%(0.05) \mathrm{n}=$ Reemplazando:

$$
\begin{array}{ll}
n= & \frac{(1.96)^{2}(0.5)(0.50)(122)}{(0.05)^{2}(122-1)+(1.96)^{2}(0.25)} \\
n=92.78=93 \text { estudiantes de Enfermería. }
\end{array}
$$

\section{Muestra reajustada}

na $=$

$$
\frac{\mathrm{n}}{1+\underline{\mathrm{n}}}
$$

Donde:

na $=$ Muestra reajustada

$\mathrm{n}=$ Muestra

$\mathrm{N}=$ Población

\section{Reemplazando:}

$$
\mathrm{na}=\frac{93}{1+\underline{93}}
$$$$
\mathrm{na}=52.7
$$

na $=53$ estudiantes de Enfermeríaç

\section{Quedando la muestra de la siguiente manera:}

\begin{tabular}{|l|c|c|}
\hline \multicolumn{1}{|c|}{ Ciclo } & Población & Muestra \\
\hline I & 40 & 17 \\
\hline III & 58 & 26 \\
\hline V & 24 & 10 \\
\hline Total & 122 & 53 \\
\hline
\end{tabular}

Fuente: Registro de DGAYRA-2019

\subsubsection{Muestreo e Instrumento de recolección de datos}

Para la elección de la muestra, se utilizó el muestreo probabilístico (Henández, 2008, p.20).

Dentro de éste, se hizo uso del muestreo aleatorio simple; en el cual, se consideraron a todos los números impares de la lista de estudiantes matriculados en cada ciclo, quienes conformaron la muestra. El instrumento de recolección de datos fue la encuesta.

\section{RESULTADOS}

Tabla 1

Relación entre el uso de redes sociales y el rendimiento académico en estudiantes de la Escuela Profesional de Enfermería, Universidad Nacional Toribio Rodríguez de Mendoza de Amazonas, Chachapoyas, 2019

\begin{tabular}{|l|c|c|c|c|c|c|c|c|}
\hline $\begin{array}{l}\text { Uso de } \\
\text { Redes } \\
\text { Sociales }\end{array}$ & \multicolumn{8}{|c|}{ Rendimiento Académico } \\
\cline { 2 - 10 } & \multicolumn{2}{|c|}{ Malo } & \multicolumn{2}{|c|}{ Regular } & \multicolumn{2}{|c|}{ Bueno } & \multicolumn{2}{|c|}{ Total } \\
\cline { 2 - 11 } & $\mathrm{fi}$ & $\%$ & $\mathrm{fi}$ & $\%$ & $\mathrm{fi}$ & $\%$ & $\mathrm{fi}$ & $\%$ \\
\hline Siempre & 14 & 26.4 & 24 & 45.3 & 0 & 0.0 & 38 & 71.7 \\
\hline A veces & 0 & 0.0 & 2 & 3.8 & 13 & 24.5 & 15 & 28.3 \\
\hline Nunca & 0 & 0.0 & 0 & 0.0 & 0 & 0.0 & 0 & 0.0 \\
\hline Total & 14 & 26.4 & 26 & 49.1 & 13 & 24.5 & 53 & 100 \\
& & & & & & & & \\
\hline
\end{tabular}

En la tabla 1, del 100\% (53) de estudiantes, el 26.4\% (14) siempre utilizan las redes sociales y tienen un rendimiento académico malo, así mismo el 45.3\% (24) siempre utilizan las redes sociales y tienen un rendimiento académico regular. Por otro lado el 3.8\% (2) a veces utilizan las redes sociales y presentan un rendimiento académico regular; sin embargo el $24.5 \%$ (13) a veces utilizan las redes sociales y tienen un rendimiento académico bueno. El análisis estadístico indica que el uso de las redes sociales influye en el rendimiento académico. $(p>0.05)$. Se puede observar también que todos los estudiantes hacen uso de las redes sociales ya sea A veces o Siempre. 


\section{Tabla 2}

Uso de redes sociales en estudiantes de la Escuela Profesional de Enfermería, Universidad Nacional Toribio Rodríguez de Mendoza de Amazonas, Chachapoyas - 2019

\begin{tabular}{ccc}
\hline $\begin{array}{c}\text { Uso de Redes } \\
\text { Sociales }\end{array}$ & fi & $\%$ \\
\hline A veces & 15 & 28.3 \\
Siempre & 38 & 71.7 \\
Nunca & 0 & 0.0 \\
\hline Total & 53 & 100.0 \\
\hline
\end{tabular}

En la tabla 2, se observa que del 100\% (53) estudiantes, el $71.7 \%$ (38) siempre hacen uso de las redes sociales, así mismo el $28.3 \%$ (15) a veces hacen uso de las redes sociales.

\section{Tabla 3}

Rendimiento Académico en estudiantes de la Escuela Profesional de Enfermería, Universidad Nacional Toribio Rodríguez de Mendoza de Amazonas, Chachapoyas - 2019

\begin{tabular}{ccc}
\hline $\begin{array}{c}\text { Rendimiento } \\
\text { académico }\end{array}$ & fi & $\%$ \\
\hline Malo & 14 & 26.4 \\
Regular & 26 & 49.1 \\
Bueno & 13 & 24.5 \\
\hline Total & 53 & 100.0 \\
\hline
\end{tabular}

En la tabla 3, se observa que del 100\% (53) estudiantes de la Escuela Profesional de Enfermería, el 49.1\% (26) de estudiantes tienen un rendimiento académico regular, el $26.4 \%$ (14) tienen un rendimiento académico malo, solo el $24.5 \%$ (13) de estudiantes presentan un rendimiento académico bueno.

\section{DISCUSIÓN}

Los resultados de la investigación demuestran que la variable uso de redes sociales tiene relación con la variable rendimiento académico en estudiantes de la Escuela Profesional de Enfermería del I al V ciclo correspondiente al semestre 2019-I, afirmando de esta manera la $\mathrm{H}_{1}$, cuyo valor del chi-cuadrado calculado fue: $\mathrm{X}^{2}=43.9$, y del chi cuadrado crítico fue: $\alpha=0.01 \mathrm{GL}: 4, \mathrm{X}^{2}=0.297$.
De igual manera Cortéz, A. (2018), Nuevo Chimbote, logró establecer la relación entre las variables; el uso de las redes sociales y rendimiento académico, a través de la correlación de Pearson, el mismo que dio como resultado un valor $\mathrm{r} 0,525$, que significa que la relación es relativamente positiva, es decir, que existe una tendencia hacia el uso de las redes sociales que puede afectar el rendimiento académico de los alumnos. Caso similar a la correlación de variables de la presente investigación.

En tal sentido, se pudo encontrar una frecuencia importante en el uso de las redes sociales, se aprecia que del 100.0\% de los estudiantes de Enfermería, el $45.3 \%$ de estudiantes siempre hacen uso de las redes sociales y tienen un rendimiento académico regular, solo el $24.5 \%$ de estudiantes que hacen uso de las redes sociales A veces cuentan con un rendimiento académico bueno. En cuanto a las horas que utilizan las redes sociales, el $32.1 \%$ de los estudiantes de Enfermería ocupan de 5 horas a más en las redes sociales; lo que nos indica que los estudiantes hacen uso de las redes sociales a diario, invirtiendo muchas horas al día sin pensar en las consecuencias que puede acarrear para su rendimiento académico y así mismo, en su futuro profesional.

Rodríguez, C. (2019), Trujillo, concluye que la frecuencia de tiempo de uso de redes sociales fue de 180 minutos en una población de 145 estudiantes del II ciclo de medicina humana. Del mismo modo afirmando que si hay relación entre el tiempo de uso de redes sociales y el rendimiento académico en los estudiantes.

Contrastando con el estudio realizado por Bustos et al. (2016), en Ecuador concluye que: el rendimiento académico de los sujetos de estudio se ve afectado por el uso de las redes sociales cuando el tiempo de acceso a estas es prolongado e indiscriminado, a pesar de que estas redes tienen un gran potencial para mejorar actividades de carácter cognitivo y educativo con la correcta supervisión de un docente. Lo que afirma los resultados de nuestra investigación.

Así mismo, podemos apreciar que el $22.6 \%$ de estudiantes de Enfermería, hacen uso de las redes sociales a veces y están en el intervalo de edad de 1620 años, un 5.7\% hace uso a veces de las redes sociales están en el intervalo de edad de 21-25 años, un $62.3 \%$ de estudiantes tienen entre 16-20 años de edad y hacen uso siempre de las redes sociales, solo el 9.4\% de estudiantes que siempre hacen uso de las redes sociales tienen de 21-25 años de edad.

En cuanto al género y uso de las redes sociales de los estudiantes de la Escuela Profesional de Enfermería, el $69.8 \%$ son de género femenino y solo el $30.2 \%$ son de género masculino, de los cuales el $50.9 \%$ y el $20.8 \%$ siempre hacen uso de las redes sociales, respectivamente. 


\section{CONCLUSIONES}

Existe relación directa y significativa entre el uso de redes sociales y el rendimiento académico de los estudiantes de Enfermería del I al V ciclo, dado que los estudiantes indicaron que usan las redes sociales con una frecuencia de siempre y a veces, ello implica que su rendimiento académico sea principalmente bajo.

Los estudiantes de Enfermería del I al V ciclo, mantienen un rendimiento académico de nivel regular a malo, con un $49.1 \%$, esto debido al uso de las redes sociales.

El 50.9\%, de estudiantes de Enfermería del I al V ciclo que hacen uso de las redes sociales son del sexo femenino y se encuentran entre las edades de 16 a 20 años de edad.

\section{REFERENCIAS BIBLIOGRÁFICAS}

Añari, A., y Valencia, B. (2017). Influencia de las redes sociales en el rendimiento escolar de los estudiantes del nivel secundario de la institución educativa 40038 Jorge Basadre Grohmann, distrito de José Luis Bustamante y Rivero, Arequipa, 2017 (tesis de pregrado). Universidad Nacional San Agustín de Arequipa, Arequipa, Perú.

Bustos, A., Flores, B., y Flores, F. (2016). Las redes sociales, su influencia e incidencia en el rendimiento académico de los estudiantes de una entidad educativa ecuatoriana en las asignaturas de Física y Matemática (tesis de pregrado). Escuela Superior Politécnica del Litoral, Guayaquil, Ecuador.

Cano, J., y Villaizan, L. (2017). Uso del Facebooky Rendimiento Académico de los estudiantes de 3 ro y 4to semestre de la facultad de Ciencias de la Comunicación (tesis de pregrado). Universidad Nacional del Centro del Perú, Huancayo, Perú.

Cortéz, A. (2018). El uso de las redes sociales y su relación con el rendimiento académico de los alumnos del cuarto grado del nivel secundario de la institución educativa $N^{\circ} 88036$ Mariano Melgar-2015 (tesis de pregrado). Universidad Nacional del Santa, Nuevo Chimbote, Perú.

Defensor del Pueblo. (2010). Programación y contenidos de la televisión e internet: la opini ón de los menores sobre la protección de sus derechos. Recuperado de https://s.libertaddigit al.com/doc/informe-del-defensor-del-pueblo-
Diario Oficial El Peruano. (2020). Facebook es la red social más popular en el Perú. Recuperado de https://elperuano.pe/noticia-facebook-es-redsocial-mas-popular-el-peru-0Twitter

Hernández, G., y Castro, A. (2014). Influencia de las redes sociales de internet en el rendimiento académico del área de informática en los estudiantes de los grados $8^{\circ}$ y $9^{\circ}$ del instituto promoción social del norte de Bucaramanga (tesis de maestría). Universidad del Tolima, Ibague, Colombia.

Hernández, R., Fernández, C., Baptista, P., Hernández, R., Fernández, C., \& Baptista, M. (2012). Metodología de la Investigación: Definición del tipo de investigación a realizar: básicamente exploratoria, descriptiva, correlacional o explicativa. México D.F., México, Mc Graw Hill.

Hernández, R. (2008). Metodología de la investigación. Ciudad de México, México. Editorial Me Graw Hill.

Ibarra, J., Hernàndez, C., Ventura, C. (2019). Hábitos alimentarios y rendimiento académico en escolares adolescentes de Chile. Revista Española de Nutriciòn humana y Dietètica, 23(4): 292-301.

IPSOS. (2020). Redes sociales en el Perú Urbano. Recuperado de https://www.ipsos.com/espe/redes-sociales-en-el-peru-urbano

Limachi, N. (2019). Uso de redes sociales y rendimiento académico de los estudiantes de la escuela de ciencias de la comunicación de la Universidad Nacional José Faustino Sánchez Carrión (2019). Universidad Nacional José Faustino Sánchez Carrión, Huacho, Perú.

Mamani, M. (2019). Influencia de las redes sociales en el rendimiento académico de los estudiantes del colegio Mariano Melgar Ayaviri, 2017 (tesis de pregrado). Universidad Nacional del Altiplano, Puno, Perú.

Marín, V., y Cabero, J. (2019). Las redes sociales en educación: desde la innovación a la investigaci ón educativa. Revista Iberoamericana de Educa ción a Distancia, 22(2), p. 25-33.

Morocco, S. (2015). Uso del Facebook y su incidencia en el rendimiento académico de los estudiantes de la Universidad Nacional del Altiplano, periodo 2014 (tesis de pregrado). 
Universidad Nacional del Altiplano, Puno, Perú.

Muñoz, R., Ortega, R., Batalla, M., López, M., Manresa, J., \& Terán, P. (2014). Acceso y uso de nuevas tecnologías entre los jóvenes deeduca ción secundaria, implicaciones en salud. Aten Primari, 46(2):77-88.

Palacios, G. (2018). Uso de las redes sociales y su relación con el desempeño académico (tesis de pregrado). Universidad San Ignacio de Loyola, Lima, Perú.

Rodríguez, C. (2019). Relación entre el tiempo de uso de redes sociales y el rendimiento académico en estudiantes de medicina humana (tesis de pregrado). Universidad Privada Antenor Orrego, Trujillo, Perú.

Saavedra, S. (2019). Impacto del uso de redes sociales sobre el rendimiento académico universitario en el 2019 (tesis de pregrado). Universidad San Ignacio de Loyola, Lima, Perú.

Sánchez, E. (2018). Uso de las redes sociales y su relación con el rendimiento académico en el primer semestre se los alumnos del grado séptimo de la Institución Educativa Tècnica de la ciudad de Ibaguè, Universidad Privada Norbert Wiener. Lima, Perù.

Supo, J. (2016). Metodología de la Investigación Cientifica: apuntes de las bases conceptuales de la investigación. 3ra Edic. Edit. Universi taria. Yanahuara. Arequipa.

Vilca, N. (2019). Influencia de las redes sociales en el rendimiento académico del VII ciclo de educación secundaria de la Institución Educativa Mariano Lino Urquieta de PuquinaMoquegua - 2018 (tesis de pregrado). Universidad Nacional de San Agustín de Arequipa, Arequipa, Perú. 